\title{
Performance of The Society of Thoracic Surgeons 2008 Cardiac Risk Models for Major Postoperative Complications after Heart Valve Surgery in a Chinese Population: A Multicenter Study
}

\author{
Chong Wang, MD,${ }^{1,2}$ Lei Jin, MD,${ }^{2}$ Fan Qiao, $M D,{ }^{2}$ Qing Xue, $M D,{ }^{2}$ Guan-xin Zhang, $M D,{ }^{2}$ \\ Lin Han, $\mathrm{MD}^{2}$ \\ ${ }^{1}$ Department of Cardiac Surgery, Shanghai Chest Hospital, Shanghai Jiaotong University, Shanghai, China; \\ ${ }^{2}$ Department of Cardiothoracic Surgery, Changhai Hospital, Second Military Medical University, Shanghai, China
}

\section{ABSTRACT}

Background: o evaluate the performance of Society of Thoracic Surgeons (STS) 2008 cardiac surgery risk scores for postoperative complications in Chinese patients undergoing single valve surgery at multicenter institutions.

Methods: From January 2009 through December 2012, 4493 consecutive patients older than 16 years who underwent single valve surgery at 4 cardiac surgical centers were collected and scored according to the STS 2008 risk scores. The final research population included the following isolated heart valve surgery types: aortic valve replacement, mitral valve replacement, and mitral valve repair. Calibration of the risk scores was assessed by the Hosmer-Lemeshow (H-L) test. Discrimination was tested by calculating the area under the receiver operating characteristic (ROC) curve.

Results: The observed incidence rate for cerebrovascular accident (CVA), renal failure (RF), prolonged ventilation (Vent), reoperation (Reop), prolonged postoperative length of stay (PLOS), and short postoperative LOS (SLOS) was $0.90 \%, 1.32 \%, 4.18 \%, 2.43 \%, 3.64 \%$, and $1.65 \%$, respectively. The predicted incidence rate for CVA, RF, Vent, Reop, PLOS, and SLOS was 0.76\%, 1.55\%, 4.94\%, 6.69\%, $3.92 \%$, and $2.54 \%$, respectively. The STS 2008 risk scores give an accurate calibration for individual postoperative risk in CVA, RF, Vent, and PLOS (Hosmer-Lemeshow: P $=.052, \mathrm{P}=.474, \mathrm{P}=.468$, and $\mathrm{P}=.712$, respectively). The area under the ROC curve of the STS 2008 risk scores for the above 4 postoperative complications were $0.714,0.724$, $0.727 \%$, and 0.713 , respectively.

Conclusion: The STS 2008 risk scores were suitable for major postoperative complications in patients undergoing single valve surgery, except for Reop and SLOS.

Received November 26, 2017; accepted May 11, 2018.

Chong Wang. and Lei fin contributed equally to this work.

Correspondence: Guan-xin Zhang, MD and Lin Han, MD, Department of Cardiothoracic Surgery, Changhai Hospital, 168 Changhai Road, Shanghai 200433, People's Republic of China; +86-21-31161765; fax: +86-21-65490979 (zhanggx97@163.com, hanlindoctor@botmail.com).

\section{INTRODUCTION}

Risk stratification plays an important role in cardiac surgical practice worldwide. During the last 2 decades, a number of excellent risk score systems have been developed for heart valve surgery, such as Veterans Administration (VA) score, European System for Cardiac Operative Risk Evaluation (EuroSCORE), Northern New England Cardiovascular Disease Study Group (NNE) score, Society of Cardiothoracic Surgeons of Great Britain and Ireland (Ambler) score, and Society of Thoracic Surgeons (STS) score [Grover 1994; Nashef 1999, 2012; Nowicki 2004; Ambler 2005; O’Brien 2009; Shahian 2009;]. These scores have been derived from large cardiovascular surgery databases. Their predictions are useful in helping determine indications for surgery, providing accurate informed consent, and monitoring the quality of surgeons and institutions.

Most predictive models have focused on operative mortality as an indicator of the quality of cardiac surgery. Only a few models evaluate other relevant outcomes as morbidity and postoperative in-hospital length of stay (LOS) or LOS in the intensive care unit [Kurki 2001, 2002; Huijskes 2003; Colak 2008]. The recent STS 2008 cardiac surgery risk models published 8 generic risk stratification models for predicting in postoperative complications after heart valve surgery [O'Brien 2009; Shahian 2009]. However, an objective evaluation of the performance of STS 2008 risk scores

Table 1. Distribution of Valve Procedures*

\begin{tabular}{lccc}
\hline Procedure & Number & Concomitant CABG (\%) & Unadjusted Mortality (\%) \\
\hline AVR & 2280 & $138(6.05 \%)$ & $49(2.15 \%)$ \\
MVR & 1484 & $65(4.38 \%)$ & $13(0.90 \%)$ \\
MVRepair & 729 & $90(12.35 \%)$ & $11(1.51 \%)$ \\
Total & 4493 & $293(6.52 \%)$ & $73(1.63 \%)$ \\
\hline
\end{tabular}

*CABG, coronary artery bypass; AVR, aortic valve replacement; MVR, mitral valve replacement; MVRepair, mitral valve repair. 
Table 2. Distribution of Risk Factors in Our Study Population versus STS 2008 Risk Scores*

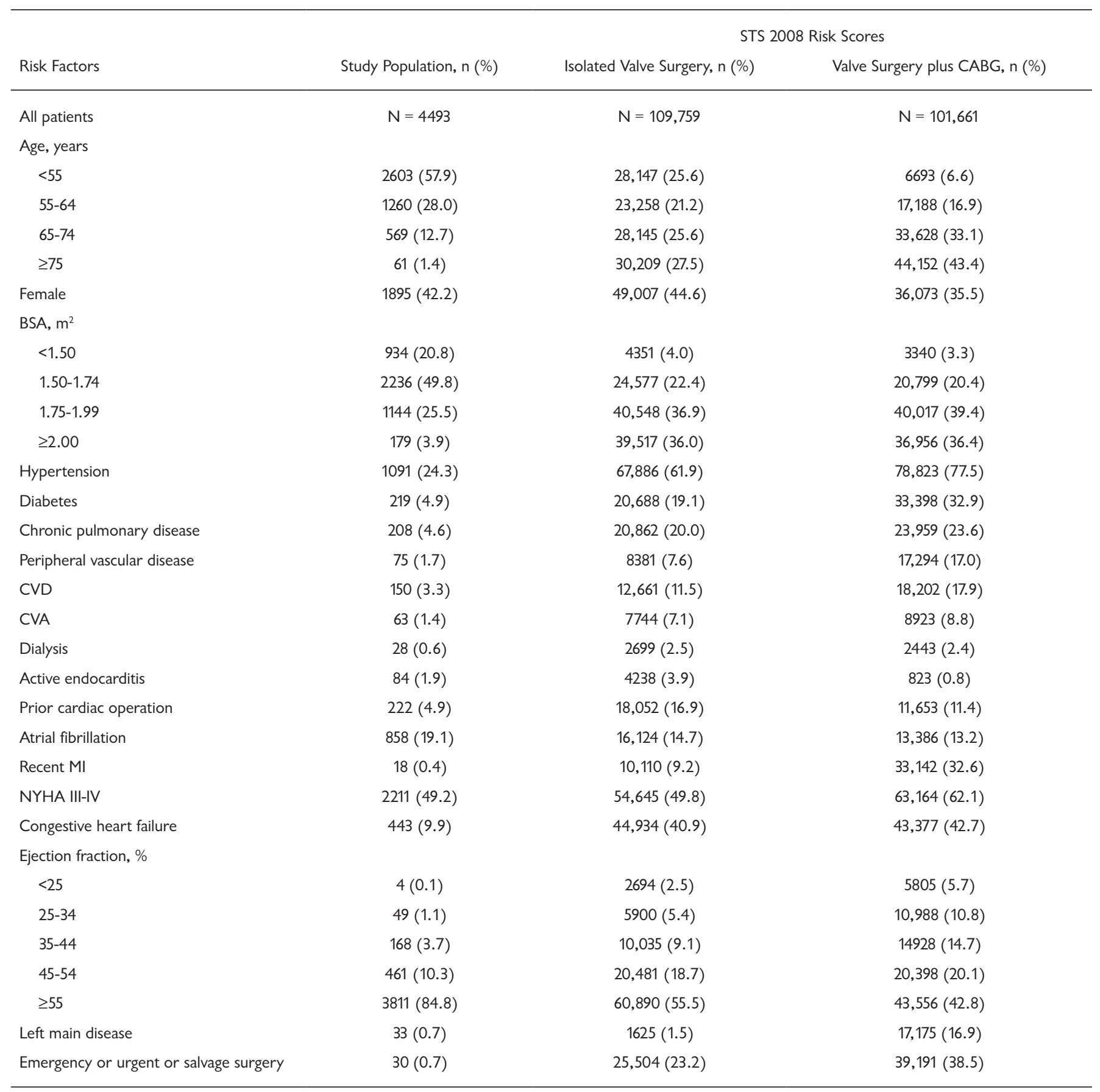

*STS, Society of Thoracic Surgeons; CABG, coronary artery bypass grafting; BSA, body surface area; CVD, cerebrovascular disease; CVA, cerebrovascular accident; MI, myocardial infarction; NYHA, New York Heart Association.

in postoperative complications after heart valve surgery in China has not been performed. Hence, the purpose of the present study was to evaluate the performance of STS 2008 risk scores in the prediction of major postoperative complications in patients undergoing heart valve surgery at multicenter institutions.

\section{MATERIALS AND METHODS}

\section{Study Population and Data Collection}

In the current retrospective study, all patients older than 16 years who underwent single valve surgery between January 2009 and December 2012 at 4 cardiac surgical centers 
Table 3. Model Calibration of the STS 2008 Risk Scores*

\begin{tabular}{|c|c|c|c|c|c|}
\hline CVA & 0.90 & 0.76 & .052 & 1.18 & 0.714 \\
\hline Vent & 4.18 & 4.94 & .468 & 0.85 & 0.727 \\
\hline Reop & 2.43 & 6.69 & $<.0001$ & 0.36 & 0.558 \\
\hline SLOS & 1.65 & 2.54 & .008 & 0.65 & 0.600 \\
\hline
\end{tabular}

*STS, Society of Thoracic Surgeons; H-L, Hosmer-Lemeshow; O/E, observed/expected; ROC, receiver operator characteristic; CVA, cerebrovascular accident (stroke); RF, renal failure; Vent, prolonged ventilation; Reop, reoperation; PLOS, prolonged length of stay; SLOS, short length of stay.

were included: Fu Wai Hospital in Beijing, Changhai Hospital of Shanghai, Zhongshan Hospital of Fudan University in Shanghai, and Guangdong Cardiovascular Institute in Guangzhou. To meet all requirements for the STS 2008 models, we included the following isolated heart valve surgery types: aortic valve replacement, mitral valve replacement, and mitral valve repair. Patients who underwent single valve surgery with concomitant tricuspid valve repair or coronary artery bypass (CABG) were also included in this study. However, valve surgery combined with aortic surgery and congenital heart procedures were excluded from the dataset.

Information on patients and the procedural risk factors for all patients were collected from the local computerized database, which was designed by the Department of Cardiothoracic Surgery, Changhai Hospital, Shanghai, China. This multicenter retrospective study was supported by Public Specialty Fund of Health Ministry (200802096). The steering committee of which includes cardiac surgeons and researchers from Changhai Hospital of Shanghai was in charge of supervision and coordination of institution recruitment and data collection. All 4 participating institutions received detailed information at the beginning of the project on

data-collection requirements and definitions of variables. The review of these records was approved by our institutional committee for human research.

Risk factors in the different risk models were selected strictly from our local database, in which all data included patient demographics, comorbidity, valve disease severity, procedural details, and outcomes. We also excluded patients who had missing information on key predictors: age, gender, operation sequence, and major postoperative complications. The predictive morbidity rate for every patient in this study was calculated by using the published STS 2008 cardiac surgery risk models [O'Brien 2009; Shahian 2009], including their risk factor definition and predictive risk calculation formula. The predicted morbidity was compared to observed morbidity for the entire cohort population.

\section{END POINTS}

The endpoints of this study were major postoperative complications referenced to STS 2008 cardiac surgery risk models, including (1) cerebrovascular accident (CVA): a central neurologic deficit persisting longer than 72 hours; (2) renal failure (RF): a new requirement for dialysis or an increase of the serum creatinine to greater than $2.0 \mathrm{mg} /$ $\mathrm{dL}$ and double the most recent preoperative creatinine level; (3) prolonged ventilation (Vent): required mechanical ventilation for 24 hours or more either continuously or in total after reintubation; (4) reoperation (Reop) for any reason; (5) prolonged postoperative length of stay (PLOS): LOS more than 14 days (alive or dead), and (6) short postoperative LOS (SLOS): LOS less than 6 days and patient alive at discharge.

\section{Statistical Analysis}

Continuous variables were expressed as mean \pm standard deviation, and categorical variables were expressed as percentages. Missing data are uncommon in our study, with a frequency of less than $1 \%$ missing for most variables. Model variables with more than $1 \%$ missing were body surface area (BSA) $(3.88 \%)$, body mass index (BMI) $(3.88 \%)$, serum creatinine $(3.11 \%)$, and left ventricular ejection fraction (LVEF) $(4.40 \%)$. To make full use of the available data, missing values were replaced by the single imputation technique [Little 2002] before the risk score was calculated.

Performance of the STS 2008 risk scores were assessed by comparing the observed and expected incidence rate for each postoperative complication. Model calibration (statistical precision) was analyzed by Hosmer-Lemeshow goodness-of-fit statistic [Hosmer 2000]. A well-calibrated model gives a $\mathrm{P}$ value greater than .05 . The calibration plot shows the mean predicted mortality against the observed mortality for 10 equally sized groups based on the ranked predicted risks calculated by the STS 2008 risk score. Model discrimination (statistical accuracy) was tested by calculating the areas under ROC curves, which was used to assess how well the model could discriminate between survivors and nonsurvivors [Hanley 1982]. The discriminative power of the model was thought to be good if the area under the ROC curve was 0.7 and very good if 0.8 . Statistical analyses were performed with IBM SPSS Statistics for Windows, version 19 (IBM Corp., Armonk, NY). 


\section{RESULTS}

\section{Patient Population and Demographics}

All 4493 patients who underwent single valve surgery procedures between January 2009 and December 2012 were evaluated for study inclusion. The distribution of valve surgeries performed are shown in Table 1. Overall, the average age $49.5 \pm 14.3$ years; $42.2 \%$ of patients were female; mean LVEF was $62.2 \% \pm 9.3 \%$; and $0.62 \%$ had emergency valve surgery. Details of the prevalence of risk factors in our populations are listed in Table 2.

\section{Model Calibration and Discrimination}

The observed incidence rate for CVA, RF, Vent, Reop, PLOS, and SLOS was $0.90 \%, 1.32 \%, 4.18 \%, 2.43 \%, 3.64 \%$, and $1.65 \%$, respectively. The predicted incidence rate for CVA, RF, Vent, Reop, PLOS, and SLOS was $0.76 \%, 1.55 \%$, $4.94 \%, 6.69 \%, 3.92 \%$, and $2.54 \%$, respectively. Table 3 shows the model calibration of the STS 2008 risk scores for 6 postoperative complications. The discriminative power (ie, the area under the ROC curve) of the STS 2008 risk scores for 6 postoperative complications are shown in Table 3 . The results mean that the STS 2008 risk scores give a better prediction for individual postoperative complications risk in patients undergoing single valve surgery, except for Reop and SLOS.

\section{DISCUSSION}

Valvular heart disease (VHD) is the most frequent acquired cardiac disease in China, and it also remains frequent in developed countries because of the predominance of degenerative valvular diseases [Nkomo 2006]. Despite an increase of average perioperative risk factors, ed or slightly reduced [Pinna Pintor 2002]. However, an increase of perioperative risk factors results in more complications after cardiac surgery and potential reduction in quality of life. Meanwhile an increase of postoperative complications led to an increase of prolonged intensive care unit (ICU) LOS and of a remarkable increase of individual costs. During the last 2 decades, several models to calculate mortality risk before heart valve surgery have been developed [Grover 1994; Nashef 1999, 2012; Nowicki 2004; Ambler 2005; O’Brien 2009; Shahian 2009]. However, most predictive models have focused on operative mortality, and a preoperative risk algorithm specifically designed to predict postoperative complications in heart valve surgery is fewer.

Of current risk prediction models for heart valve surgery, the STS 2008 risk scores had been highly successful and used worldwide in the daily clinical activities. In our previous clinical researches that were designed during a multicenter study [Wang 2014], we have analyzed the predictive value of the STS 2008 risk scores for predicting in-hospital mortality in patients undergoing heart valve surgery. However, the performance of the STS 2008 risk scores for predicting postoperative complications after heart valve surgery in China has not been clear. Therefore, this present study was designed to evaluate the performance of STS 2008 risk scores in the prediction of major postoperative complications in patients undergoing heart valve surgery at multicenter institutions.
Our study population was selected from 4 representative institutions located in the developed area of China, and the population was drawn from approximately half of the area of China. In our study, the STS 2008 risk scores give a better prediction for individual postoperative complications risk in patients undergoing single valve surgery, except for Reop and SLOS. The reasons are likely to be multifactorial. First, the candidate predictor variables were selected relatively completely by the STS 2008 risk scores for single valve surgery. Some valve disease-specific risk factors are included in the STS score development, such as valve pathological changes, which is related to the operative treatment of valvular heart disease [Nowicki 2005]. Meanwhile, the model design and statistical analysis method of predictor variables in the STS 2008 risk scores were different, and 8 generic risk stratification models for postoperative complications were designed, and interaction terms with 2 candidate variables were first used in the STS 2008 risk scores development [O'Brien 2009; Shahian 2009]. Therefore, it is not surprising that the STS 2008 risk scores gives a good calibration and discriminative power. Second, the baseline of the prevalence of risk factors in our population differed from those of the United States. The population included in our study was younger, included a greater proportion of females, had less chronic lung disease, peripheral vascular disease, renal dysfunction, coronary artery disease, recent myocardial infarction, and emergency surgery, and had higher active endocarditis, atrial fibrillation, and pulmonary hypertension at the time of valve surgery [Wang 2014]. Moreover, different risk factors selected, different weighting of these risk factors, variable patient characteristics in the initial derivation cohort, and change of patient characteristics and operative techniques could influence their accuracy and performance.

As a retrospective investigation, the incorrect and missing data in the original medical records could not be re-collected, and the definitions of risk factors are not the same in a different risk score system; this difference could affect the result of the risk algorithm.

\section{CONCLUSIONS}

The STS 2008 risk scores were suitable for major postoperative complications in patients undergoing single valve surgery, except for Reop and SLOS.

\section{REFERENCES}

Ambler G, Omar RZ, Royston P, Kinsman R, Keogh BE, Taylor KM. 2005. Generic, Generic, simple risk stratification model for heart valve surgery. Circulation 112:224-31.

Colak Z, Segotic I, Uzun S, Mazar M, Ivancan V, Majeric-Kogler V. 2008. Health related quality of life following cardiac surgery: correlation with EuroSCORE. Eur J Cardiothorac Surg 33:72-6.

Grover FL, Johnson RR, Shroyer AL, Marshall G, Hammermeister KE. 1994. The Veterans Affairs Continuous Improvement in Cardiac Surgery Study. Ann Thorac Surg 58:1845-51.

Hanley JA, McNeil BJ. 1982. The meaning and use of the area under a receiver operating characteristic (ROC) curve. Radiology 143:29-36. 
Hosmer DW, Lemeshow S. 2000. Applied logistic regression. New York: John Wiley and Sons.

Huijskes RV, Rosseel PM, Tijssen JG. 2003. Outcome prediction in coronary artery bypass grafting and valve surgery in the Netherlands: development of the Amphiascore and its comparison with the Euroscore. Eur J Cardiothorac Surg 24:741-9.

Kurki TS, Hakkinen U, Lauharanta J, Ramo J, Leijala M. 2001. Evaluation of the relationship between preoperative risk scores, postoperative and total length of stays and hospital costs in coronary bypass surgery. Eur J Cardiothorac Surg 20:1183-87.

Kurki TS, Jarvinen O, Kataja MJ, Laurikka J, Tarkka M. 2002. Performance of three preoperative risk indices; CABDEAL, EuroSCORE and Cleveland models in a prospective coronary bypass database. Eur J Cardiothorac Surg 21:406-10.

Little RJA, Rubin DB. 2002. Statistical analysis with missing data. 2nd ed. Hoboken (NJ): Wiley-Interscience.

Nashef SA, Roques F, Michel P, Gauducheau E, Lemeshow S, Salamon R. 1999. European system for cardiac operative risk evaluation (EuroSCORE). Eur J Cardiothorac Surg 16:9-13.

Nashef SA, Roques F, Sharples LD, et al. 2012. EuroSCORE II. Eur J Cardiothorac Surg 41:734-44.
Nkomo VT, Gardin JM, Skelton TN, Gottdiener JS, Scott CG, Enriquez-Sarano M. 2006. Burden of valvular heart diseases: a population-based study. Lancet 368:1005-11.

Nowicki ER, Birkmeyer NJ, Weintraub RW, et al. 2004. Multivariable prediction of in-hospital mortality associated with aortic and mitral valve surgery in northern New England. Ann Thorac Surg 77:1966-77.

Nowicki ER. 2005. What is the future of mortality prediction models in heart valve surgery? Ann Thorac Surg 80:396-98.

O'Brien SM, Shahian DM, Filardo G, et al. 2009. The Society of Thoracic Surgeons 2008 cardiac surgery risk models: part 2-isolated valve surgery. Ann Thorac Surg 88(1 suppl):S23-42.

Pinna Pintor P, Colangelo S, Bobbio S. 2002. Evolution of case-mix in heart surgery: from mortality risk to complication risk. Eur J Cardiothorac Surg 22:920-26.

Shahian DM, O'Brien SM, Filardo G, et al. 2009. The Society of Thoracic Surgeons 2008 cardiac surgery risk models: part 3-valve plus coronary artery bypass grafting surgery. Ann Thorac Surg 88(1 suppl):S43-62.

Wang L, Lu FL, Wang C, Tan MW, Xu ZY. 2014. Society of Thoracic Surgeons 2008 cardiac risk models predict in-hospital mortality of heart valve surgery in a Chinese population: a multicenter study. J Thorac Cardiovasc Surg 148:3036-41. 